\title{
Evolving Robot Behaviour at Micro (Molecular) and Macro (Molar) Action Level
}

\author{
Michela Ponticorvo ${ }^{1}$ and Orazio Miglino ${ }^{1}{ }^{2}$ \\ 1 Department of Relational Sciences "G.Iacono", University of Naples "Federico II", \\ Naples, Italy \\ ${ }^{2}$ Institute of Cognitive Sciences and Technologies, National Research Council, Rome, \\ Italy
}

\begin{abstract}
We investigate how it is possible to shape robot behaviour adopting a molecular or molar point of view. These two ways to approach the issue are inspired by Learning Psychology, whose famous representatives suggest different ways of intervening on animal behaviour.

Starting from this inspiration, we apply these two solutions to Evolutionary Robotics' models. Two populations of simulated robots, controlled by Artificial Neural Networks are evolved using Genetic Algorithms to wander in a rectangular enclosure. The first population is selected by measuring the wandering behaviour at micro-actions level, the second one is evaluated by considering the macro-actions level. Some robots are evolved with a molecular fitness function, while some others with a molar fitness function. At the end of the evolutionary process, we evaluate both populations of robots on behavioral, evolutionary and latent-learning parameters.

Choosing what kind of behaviour measurement must be employed in an evolutionary run depends on several factors, but we underline that a choice that is based on self-organization, emergence and autonomous behaviour principles, the basis Evolutionary Robotics lies on, is perfectly in line with a molar fitness function.
\end{abstract}

\section{Introduction}

Designing mobile robot's behaviour is far from trivial: designing them by hand from scratch is a very difficult task for humans, while designing them automatically doesn't assure to scale up to complicated tasks. A lot of energy in this process is devoted to behaviour description. We usually describe the behaviour we wish to model in qualitative terms that are then translated in the equation required for automatic evaluation. But, in doing this, we can assume various points of view, different analysis levels. For example, if we want a robot to play soccer, to follow the shortcut up to a target or just to wander in an environment without sticking into obstacles, we can concentrate on constraints on a microlevel (the sensors or motors state) or on global behaviours (score goals or explore an arena). This latter frame of description is the one assumed, for instance, by Behavior Based Robotics [1], an approach in which the desired behaviour is divided into a set of simpler behaviours. With this approach, however, the designer 
must decide which are these simpler behaviours. Also in Robot Shaping [3], a technique that deals with designing and building learning autonomous robots in which the human designer proposes how a task should be carried out and how it should be decomposed into sub-tasks that are then implemented at the robot level and reinforced, the focus is on behaviour level.

The decision about behaviour description to be kept for robot behaviour is not different from what must be defined in the supervision of learning in natural organisms. In fact we talk about shaping, a term coming from experimental psychology [19] where it describes a particular technique to train animals. In other words, Robot Shaping, Behavior Based Robotics and, as we will see, also other techniques for robot behaviour design, must face the same problem that encountered Behaviorist Psychology and encounters Learning Psychology, that is to choose the right analysis level that allows to evaluate the efficiency of training procedures on animals and human beings. What does an experimenter (but also a teacher or a breeder) must concentrate on to improve learning? This is a question many psychologists have tried to answer, proposing different interpretations with their experimental or clinical work. Hill[7]in his work on Learning Psychology has distinguished these potential solutions into two families: molecular vs. molar. What does this means?

Molecular and molar are two words derived from chemistry. The first one refers to molecules, "the smallest unit into which a substance can be divided without changing its chemical nature" (Oxford Wordpower Dictionary), while the second one refers to mole, the number of grams of a certain molecule that is equal to the number that indicates its molecular weight. More generically we can consider a mole a set of various molecules of the same kind. In Learning Psychology these terms are adopted to indicate theories that consider the smallest components of a behaviour such as a muscle that moves (molecular) or the behaviour as a whole(molar), for example going out of a maze.

Some psychologists, the molecular approach supporters, believed that the way to follow was to concentrate on micro-behaviours that formed animal's performance (Pavlov [16] for aspects related to micro-actions stabilization and Guthrie $[4,5]$ for the importance to focus on micro-actions to understand behaviour). On the contrary the molar approach (cfr.Tolman[17]) preferred to reinforce macrobehaviours that lead to a satisfying final outcome, for example to localize a target area in a complex labyrinth.

These two approaches are indeed complementary: we can indeed imagine "molar" and "molecular" not as dichotomous entities, but as the extremes of a continuum for behaviour definition that may be applied to Robotics and robots'design too, as it is represented in figure 1 for a wandering behaviour. This distinction is echoed in Evolutionary Robotics, the technique we have used in the experiments described in this paper.

Evolutionary Robotics $[15,6]$ is a discipline belonging to Artificial Life [8] whose goal is to obtain artificial agents, both physical or simulated. This methodology is inspired by darwinian selection, according tho which only fittest organisms can reproduce. To run an artificial evolutionary process according to Evolutionary 


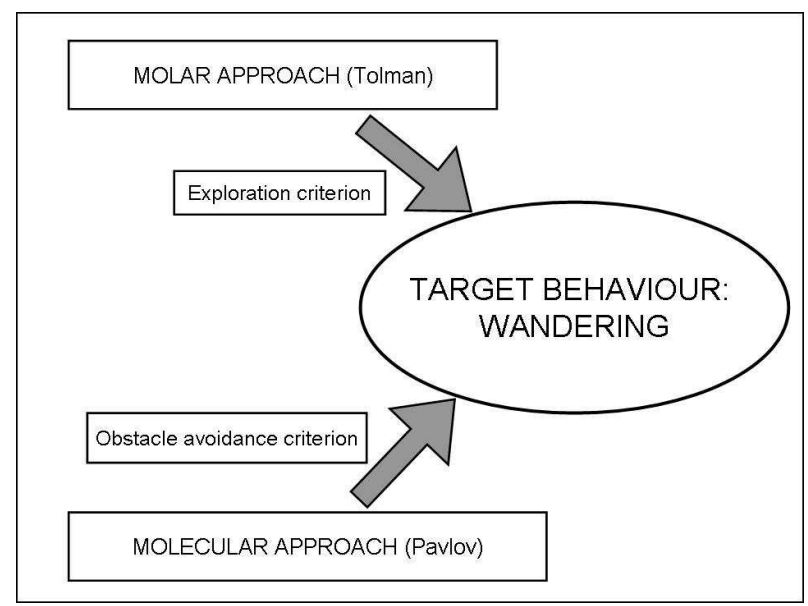

Fig. 1. Two ways to produce a wandering behaviour: we can described it at the lower level of a set of micro-actions (measures of sensors and motors activation or internal states) in the molecular approach or at the level of macro-actions in the molar approach.

Robotics' dictates it is necessary to define a criterion that addresses the entire evolution. A typical experiment in Evolutionary Robotics can be described like this: an initial population of robots whose features are defined in an artificial genotype, is tested and evaluated according to a criterion, usually referred to as the fitness function. For example we may define in the genotype the connections' weights of an Artificial Neural Network, the robot controller that determines the behaviour. The robots that obtain the best scores are allowed to reproduce: their genotypes, opportunely mutated or crossed, will constitute the genotypes of the second generation and will thus determine the second generation phenotypes and behaviours. The testing-evaluating-reproducing loop is iterated for a certain number of generations or until at least some robots display the desired behaviour or solve the predefined task. It is clear from this brief description the fundamental role played by the fitness function in the evolutionary process, as this function is used to evaluate the performance of robots and to select the ones that will reproduce. In fact robots are evaluated about their ability according to the criterion defined by the experimenter, measured by the fitness function. The probability that a robot reproduces depends on the score obtained in respect to this function. Consequently fitness formula's design is fundamental in every Evolutionary Robotics'experiment.

This aspect has been underlined since the seminal work by Nolfi and Floreano [15] who proposed a framework for describing various fitness functions: the Fitness Space. This fitness space is a three-dimensional space with axes representing three continuous dimensions that are relevant for fitness functions. A fitness function can then be imagined as a point in this space.

The first dimension goes from "functional" to "behavioral". A functional fitness function focuses on functioning modes of the controller, while a behavioral one 
evaluates its behavioral outcome.

The second dimension "explicit vs.implicit" considers the amount of constraints that are taken into account in the fitness function. An explicit fitness function considers many precise components, while an implicit one has just few constraints or not at all.

The latter dimension has "external" and "internal" as extremes, indicating if the variables in the fitness function are accessible to the evolving robot computation. In an internal fitness function variables are calculated on sensors' activation of the robots, while in an external one on information available only for the experimenter.

Choosing a fitness function is not a trivial decision that strictly depends on the purpose of the evolutionary process and will address the whole bulk of results. Also in this case, it is necessary to find the right mix between molar and molecular. For example, when Nolfi and Floreano [15] want to obtain an obstacle avoidance behaviour, they use a molecular fitness function that rewards particular micro-actions(we will describe it in detail in the next section). If, on the contrary, the task is to localize an area inside an arena, it's not compulsory to consider micro-actions and it can be more useful to use a molar function [9]. With more complex tasks, integration of the two approaches may be a right choice [12]. Another example of differently conceived fitness functions can be found in co-evolution experiments. To study prey-predator dynamics Nolfi and Floreano [14] use a molar fitness function that assigns simply 1 point for the predator and 0 for the prey if the predator is able to catch the prey and 0 for the predator and 1 for the prey if it escapes the predator. On the contrary Cliff and Miller [2] used in their experiments on co-evolution a more complex fitness function that includes more constraints that address evolution, for example predators are also scored for their ability to approach the prey.

So it seems to us very fruitful to analyze the possible outcome of differently conceived fitness functions as it might shed light on how to guide learning processes. In this paper this is what try to do, that is to compare two differently characterized fitness functions (molecular vs. molar) on the same behaviour to verify what happens at behavioral, learning or evolutive level.

\section{Wandering in a closed arena without sticking into obstacles: two possible fitness functions}

The task we have tested the fitness functions on is a simple wandering task. We just want the robot to move in a rectangular enclosure without bumping into walls and into cylindric obstacle that are inside the arena.

In order to compare two differently conceived fitness functions we analyze the molecular fitness function used by Nolfi and Floreano [15] to obtain an obstacle avoidance behaviour and a molar one proposed by Walker and Miglino [20]. The molecular fitness function is composed by three distinct components that reward three variables: the average of robot wheels' speed, the wheels'differential and the activation value of the most active infrared sensor. These three components 
encourage motion, straight movement and obstacle avoidance.

For the molar fitness function we divide the rectangular arena into 50 cells $\left(10^{*} 5\right)$ and we give the robot a reward that corresponds to the number of cells visited for the first time.

\section{Method}

In our experiments, we run two different evolutionary process to obtain a wandering behaviour. In the first one the population of robots is selected with the molecular fitness function by Nolfi and Floreano [15] described in the previous section. In the second one the robots are selected using the molar fitness function. We use the EvoRobot simulator [13] to run the evolutionary process on the software robots.

\subsection{Robots}

Each robot consists of a physically accurate simulation of a round robot, with a diameter of $5.5 \mathrm{~cm}$. Each robot is equipped with 8 infrared proximity sensors (capable of detecting objects within $3 \mathrm{~cm}$ of the sensor). The robots move using 2 wheels (one on each side of the robot) powered by separate, independently controlled motors. The control system is an Artificial Neural Network: a perceptron whose input layer is formed by 8 input units that codify the activation of Infrared Sensors that receive stimulation from obstacles up to $5 \mathrm{~cm}$. In the output layer there are 2 output neurons, totally connected to all input units, that control wheels.

\subsection{Training environment}

In our experiments, we use this training environment: a rectangular arena $(500 * 1000$ $\mathrm{mm}$.) in which there are 5 obstacle in randomly chosen position. These obstacles are cylinders with $27.5 \mathrm{~mm}$. radius.

\subsection{Training procedure}

The robots are trained using a Genetic Algorithm [10].

At the beginning of each experiment, we create 100 simulated robots with random connection weights. We then test each robot's ability to wander inside the arena. The robot is positioned in a random location and allowed to move around for 100 computation cycles (1ms per cycle). The robots are rewarded according to the two fitness functions described before: a molar and a molecular one. At the end of this procedure, the 80 robots with the lowest scores are eliminated (truncation selection). The remaining 20 robots are then cloned (asexual reproduction). Each "parent" produces five "offspring". During cloning, 25 per cent of neural connections are incremented by random values uniformly distributed in the interval $[-1,+1]$. The testing/selection/cloning cycle is iterated for 100 "generations". For each population the simulation is repeated twenty times with the same parameters and randomly generated initial connecting patterns. 


\section{Results}

\subsection{Evolutionary patterns}

In figure 2 and 3 the evolutionary trends for the two populations are represented.

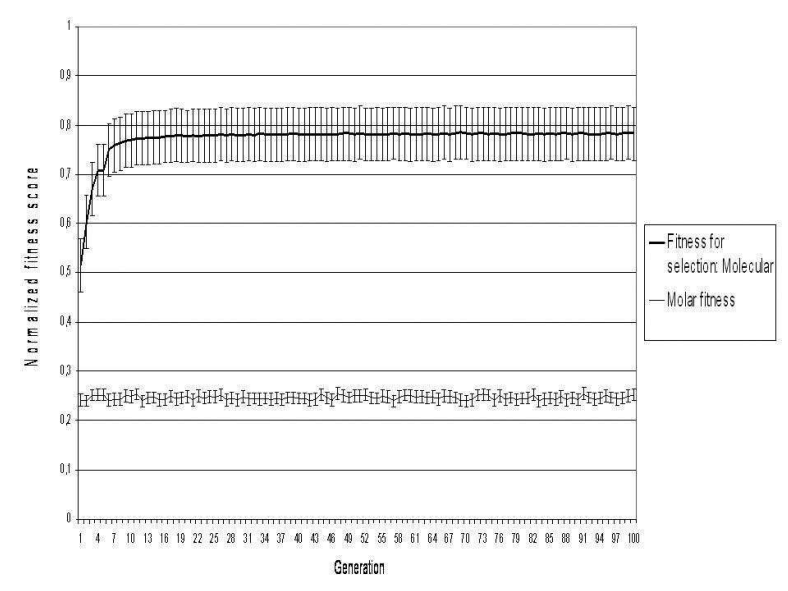

Fig. 2. Evolutionary trend for robots selected by the molecular fitness function. On $x$ axis there are generations, on y axis fitness scores. The thin line indicates the score gained on the molar fitness function, that is not considered for selection.

The scores regard the average of the best robots of each seed along generations. As it can be seen standard deviation is lower for the molar fitness function. In the figures above the scores on the fitness function not used for evolution are represented, as these data are relevant for another analysis we will describe in the following section.

\subsection{Training speed}

We take then into account an evolutionary parameter that is the time lapse from initial generation up to the peak of fitness. This is a measure of evolutionary process speed, a parameter that is crucial for Evolutionary Robotics, as robot evaluation may require a long time. It is also an indirect measure of how "easy" a task is for the evolving robot. If less time is required to reach the maximum in terms of fitness scores, this means that the task is easier to accomplish for the system formed by the robot and the environment that constraints its action. We thus compare the emergence time of fitness peaks in the two populations of robots. We observe that for the robots evolved with the molar fitness function the best solution appears after less generations than for the molecular fitness function. This difference is statistically significant.

$$
t(38)=6,41 ; p=0,00 .
$$




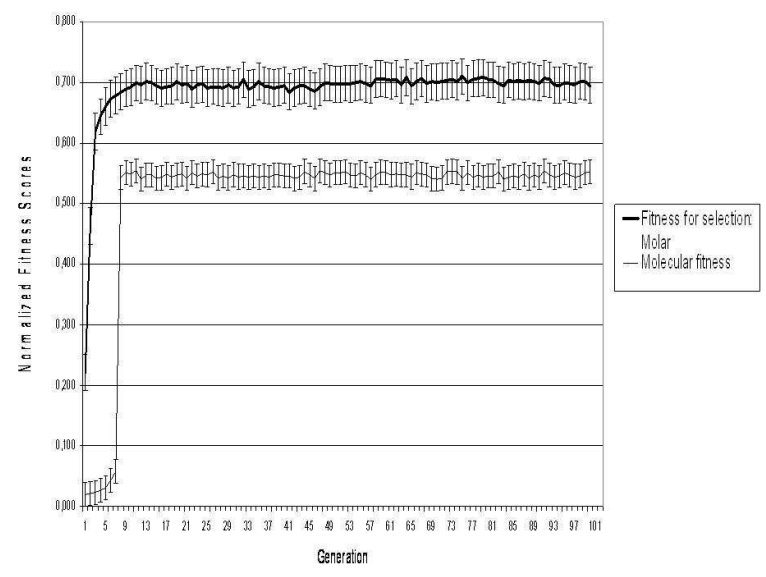

Fig. 3. Evolutionary trend for robots selected by the molar fitness function. On x axis there are generations, on y axis fitness scores. The thin line indicates the score gained on the molecular fitness function, that is not considered for selection.

\subsection{Fitness functions comparison}

We compare the two fitness function on what we call latent learning. This is a concept close to the one of generalization, but it is more focused on generalization lying inside a certain task. This means that we wonder if, during the evolutionary process, robots learn something else about the task that is not explicitly rewarded. In this case we would like to know if, while evolved for wandering with a molar function they maximize also the constraints of the molecular one and vice-versa. From figures 2 and 3 we can infer that robots evolved with the molar fitness function gain good scores in the molecular one, but robots evolved with the molecular fitness function score bad on the molar one. So we run another test on this issue: what happens if we evaluate the best 20 robots evolved with the molar fitness function using the molecular one? The fitness scores, even if less high in average than the corresponding values obtained with molecular fitness function, are not significantly different.

$$
t(38)=0,95 ; p=0,34 .
$$

What happens if, on the contrary, we go the other way around, testing the best 20 robots evolved with molecular fitness function using the molar one? The results obtained by these robots are significantly lower than the ones obtained with the molar function.

$$
t(38)=9,92 ; p=0,00 .
$$

This means that the molar fitness function, even if not explicitly designed to maximize the three components that form the molecular one, "contains" nevertheless these variables implicitly and addresses the evolutionary process in order 
to maximize them. On the contrary, the molecular fitness function does not allow the emergence of the exploratory behaviour rewarded by the molar function. This means that the molar fitness function "includes" the molecular one in terms of latent learning but not vice-versa.

\subsection{Behavioral Analysis}

What happens at the behavioral level? Figures 4 and 5 show the trajectories by two very efficient robots from the populations evolved with molecular and molar fitness functions.

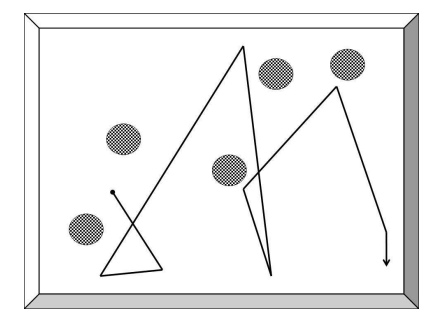

Fig. 4. Behaviour displayed by an individual evolved with the molecular fitness function.

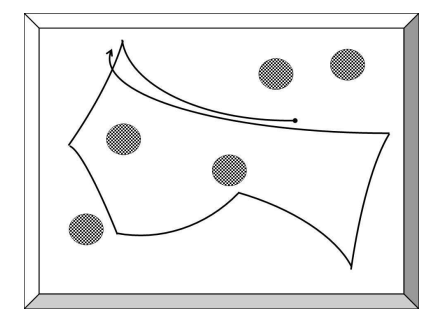

Fig. 5. Behaviour displayed by an individual evolved with the molar fitness function.

As it can be seen from the figures above, the behaviours displayed by robots evolved with different fitness functions are quite different. The robots evolved with the molecular fitness function proceed straight until they reach a wall or an obstacle, at this point they turn left or right, avoid the obstacle and continue their run.

On the other side, robots that have been evolved with the molar fitness function proceed fast and avoid obstacle but, in many cases they do not go straight. In fact between these robots we can find, together with some agents that displace 
straightly, many robots that follow curved trajectories, drawing curves with different radius and avoiding obstacles when encountered. The behaviours that emerge with the molar fitness function are much more varied.

\section{Conclusions}

The data exposed above suggest that a wandering behaviour can emerge adopting both a molar and a molecular point of view. Why should we prefer one or the other? As happens in natural organisms' learning supervision, it depends on what we want to achieve. The molecular function can be the right choice in some cases and the molar function in some others.

The presented results lead us to sustain, for the simple task we analyzed, the molar one. Why? It allows the system to build its own solution freely. Regardless of what an experimenter thinks a solution should be like in details, a global solution emerge by itself. This is possible because the system formed by the robot and the environment can self-organize, thus exploring ways to solution that may be not considered a priori. What does this emergent solution look like? First of all it includes a wider set of strategies.

To explore an environment while avoiding obstacles we could believe that the best is to go straight, but following curved trajectories may be a good way to solve the same task. This kind of solution, that does not emerge in the case of the molecular fitness function, is equally efficient and enriches the whole of solutions between which evolution can look for. This is surely an advantage in an evolutionary perspective. With this kind of function, robots are favored to establish a useful relation with the environment exploiting a very precise coordination between input and output, thus adapting to external constraints.

Good hints can be found at the evolutionary level too: the evolutionary process appears to be faster with the molar function as the fitness peak is reached in fewer generations. Moreover the standard deviation in fitness scores is low, thus indicating that a great amount of robots is able to obtain high fitness values. Another positive aspects is about latent learning. The molar function permits to improve also the scores on the molecular constraints, even if not explicitly considered. In other words, it shows a good latent learning. The last, but not least, reason has been already partly discussed and is about the preference for a self-organizing solution. Letting the system the possibility to self-organize, unexpected and more efficient solutions can emerge, also suggesting new ways of approaching a certain problem, an important issue in scaling to more complex behaviours.

In fact, in building an intelligent robot a mechanical creature which can function autonomously" (pag.3), that is one of to purpose of Artificial Intelligence, the science of making machines act intelligently(pag.15) [11], we must keep in mind that autonomy is fundamental, also in the sense that the robot must operate without human-intervention, supervision or instruction and adapt to changes in the environment and itself. If this is the goal, also the means to reach it should be informed to the maximum possible autonomy, what we can do choosing a 
molar fitness function in Evolutionary Robotics rather than a molar one.

In closing we would like to underline that, even if these results do not apply directly to natural organisms' learning supervision, they nonetheless supply interesting insights on this controversial issue.

\section{References}

1. Brooks, R.A.: Intelligence without representation. Artificial Intelligence (47), 139159 (1991)

2. Cliff, D., Miller, G.F.: Tracking the read queen: Measurement of adaptive progress in coevolutionary simulations. In F. Moran, A. Moreno, J.J. Merelo, P. Chacon (eds.): Advances in Artificial Life: Proceedings of the Third European Conference on Artificial Life, Berlin:Springer Verlag (1995)

3. Dorigo, M., Colombetti, M.: Robot Shaping. An Experiment in Behavior Engineering. Intelligent Robotics and Autonomous Agents series, vol. 2. MIT Press (1998)

4. Guthrie, E.R.: The psychology of learning. Gloucester, MA: Simith (1960)

5. Guthrie, E.R., Horton, G.P.: Cats in a puzzle box. New York: Rinehart (1946)

6. Harvey, I., Husbands, P., Cliff, D., Thompson A., Jakobi N. Evolutionary robotics: The Sussex approach. Robotics and Autonomous Systems, 20:205-224(1997)

7. Hill, W.F.: Learning: A survey of psychological interpretations. Paperback (1973)

8. Langton, C.G.: Artificial Life: An Overview.Cambridge, MA: The M. I. T. Press/A Bradford Book (1995)

9. Miglino, O., Lund, H.H.: Do rats need euclidean cognitive maps of the environmental shape? Cognitive Processing, 1-9(2001)

10. Mitchell, M.: An Introduction to Genetic Algorithms. Cambridge, MA: MIT Press(1996)

11. Murphy, R. R.: Introduction to AI robotics. Cambridge, MA: MIT Press/Bradford (2000)

12. Nolfi, S.: Evolving non-trivial behaviors on real robots: a garbage collecting robot. Robotics and Autonomous System, 22: 187-198(1997)

13. Nolfi, S. (2000). Evorobot 1.1 User Manual. Technical Report, Institute of Psychology, Rome

14. Nolfi, S., Floreano, D.: How co-evolution can enhance the adaptive power of artificial evolution: Implications for evolutionary robotics. In P. Husbands and J.-A. Meyer (Eds.), Proceedings of the First European Workshop on Evolutionary Robotics, Berlin: Springer Verlag, pp.22-38 (1998)

15. Nolfi, S.,Floreano, D.: Evolutionary Robotics: The Biology, Intelligence and Technology of Self-Organizing Machines. Cambridge, MA: MIT Press/Bradford (2000)

16. Pavlov, I.P.: Conditioned reflexes. Oxford University Press (1927)

17. Tolman, E.C.: Purposive behavior in animals and men. New York: AppletonCentury-Crofts (1932)

18. Sharkey, N.E., Heemskerk, J.: The neural mind and the robot, In A. J. Browne (Ed.), Neural Network Perspectives on Cognition and Adaptive Robotics, Bristol U.K. :IOP press (1997)

19. Skinner, B.F.: The behavior of organisms: An experimental analysis, New York: Appleton-Century-Crofts (1938)

20. Walker, R., Miglino, O.: Simulating exploratory behavior in evolving Artificial Neural Networks. Proceedings of Gecco1999, Morgan Kauffman (1999) 